\title{
Simulation and Evaluation of Pulsed Phase Thermography for the Quality Control of Resistance Spot Welds
}

by U. Siemer*

*Volkswagen AG, Wolfsburg, Germany

\begin{abstract}
Since pulsed phase thermography is establishing itself as testing method for laser weld inspection, this non-destructive thermal technique becomes increasingly attractive for the quality control of joints in automotive body structures. This work concentrates on the measurement of the diameter of resistance spot welds and its disturbances which need to be suppressed. Three-dimensional finite element models of typical spot weld geometries are developed and validated using both model and experiment. They allow to describe the distribution of the idealized transient surface temperature corresponding to the inner heat transfer of the sample. An adequate way to test the robustness of analysis algorithms and first results of recent studies are presented.
\end{abstract}

\section{Introduction}

To evaluate the joining quality of automotive body structures, there are still many costly destructive testing methods in use. Approved techniques and state of the art for process control of resistance spot welds are destructive methods like chisel testing and metallographic examinations (microsections). Using a reliable nondestructive testing method ensuring the joint quality, the quality management and scrap costs could be reduced considerably. For a restricted application range of laser welded car bodies, thermographic pulse techniques with flash light excitation are successfully implemented by now. This represents a reliable testing method for a half automated sampling inspection sufficiently robust for the utilization under production line conditions. To pursue these progresses in laser weld testing it is intended to enhance these potentials particularly with regard to resistance spot weld evaluation.

Several investigations describe the ability of thermal techniques to characterize spot welds $[1,2,3]$, but a successful adaptation to production line requirements has not been realized yet. To provide thermographic equipment capable of replacing recent cost intensive destructive testing procedures, questions concerning the evaluation criteria, adequate stimulation methods and sufficiently robust data processing techniques have to be clarified more comprehensively. Some prior publications indicate for example the feasibility to identify incomplete or fragile welds (characterized by solid but weak contact) [1,2] and mention the possibility to estimate spot weld sizes [3]. In most cases, however, they are restricted to specific conditions (e. g. uncoated plates, black painted surfaces, through transmission configuration). The primary issue of this work is to determine the weld size as the most important quality criterion. The experimental setup, suitable for industrial use, is instrumented with a pulsed excitation in a reflection mode, i.e. the camera is on the same side of the specimen as the heat source. The considered samples are spot welded steel sheet material common for automotive body structures.

The investigations are supported by finite element calculations in order to provide useful information about the expected thermal response of the sample in the 
experiment. This can be used to optimize the data processing algorithms and helps to quantify the thermographic output for weld size prediction. Also an estimation of the testing feasibilities will be possible in an early development stage and worst case situations can be taken into account. The following figure shows the interaction between the experiment and the supporting simulation model to define the optimization potentials.
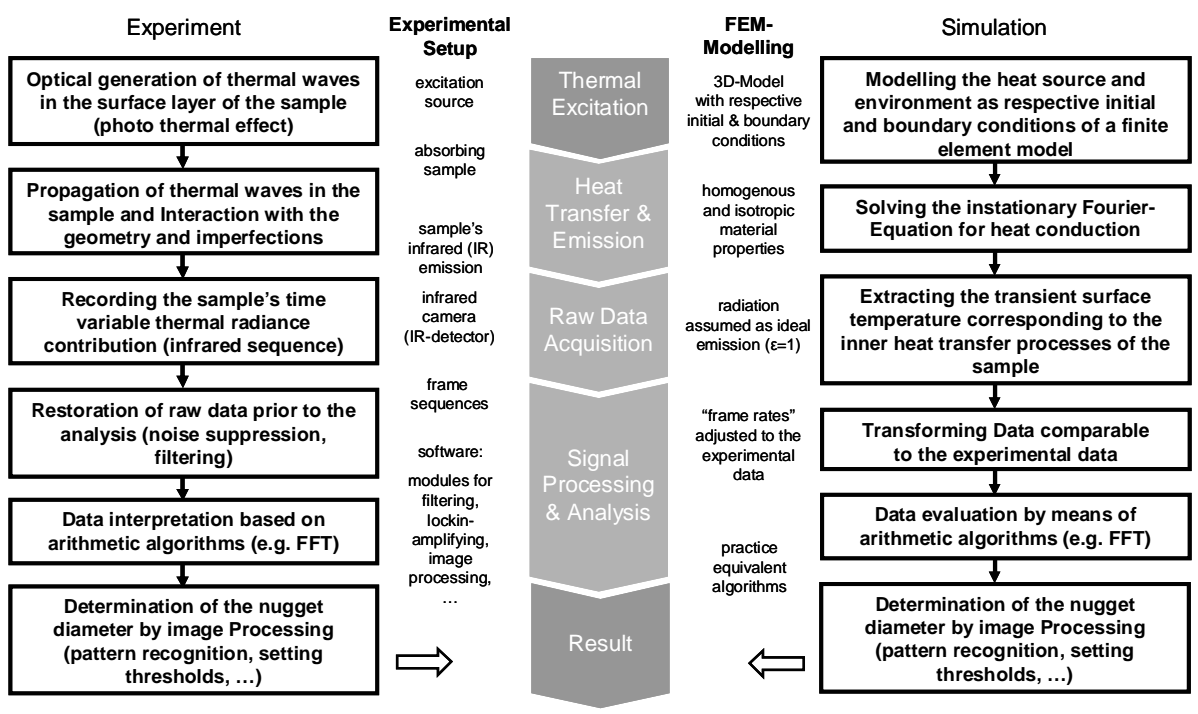

Figure 1. Experiment and Simulation Duality of Considerations

\section{Fundamentals of Non-Destructive Thermographic Testing}

\subsection{Thermal Wave Theory for Thermograpy}

If modulated optical radiation hits a solid surface of a semi-infinite sample, it will be partly reflected, absorbed or transmitted. Due to the photothermal effect the absorbed fraction of the incident electromagnetic waves launches a heat front within a thin surface layer depending on optical and thermal properties of the material under investigation $[4,5]$. Thus, highly damped thermal waves disperse into the material [6] with the propagation speed $v_{p}$,

$$
v_{p}=\sqrt{2 \alpha \omega}
$$

which depends on their modulation frequency $\omega=2 \pi f[\mathrm{rad} / \mathrm{s}]$ with the frequency $f[\mathrm{~Hz}]$ and the material's thermal diffusivity $\alpha=\lambda / \rho c\left[\mathrm{~m}^{2} \mathrm{~s}^{-1}\right]$ with the thermal conductivity $\lambda$ $\left[\mathrm{W} /\left(\mathrm{m}^{*} \mathrm{~K}\right)\right]$, the density $\rho\left[\mathrm{kg} / \mathrm{m}^{3}\right]$ and the specific heat $c\left[\mathrm{~J} /\left(\mathrm{K}^{*} \mathrm{~kg}\right)\right]$. Caused by the attenuation a thermal wave has a specific diffusion length $\mu$ expressed as a function of the modulation frequency and the thermal diffusivity as well: 


$$
\mu=\sqrt{2 \alpha / \omega}
$$

and the wavelength is defined as:

$$
\lambda=2 \pi \mu
$$

The general description of the thermal wave propagation in 3-dimensional solids is given by the following transient differential equation of heat conduction called Fourier Equation [7,4]:

$$
\rho(\vec{r}, T) c(\vec{r}, T) \frac{\partial T(\vec{r}, t)}{\partial t}=\lambda_{\vec{r}, T} \frac{\partial^{2} T(\vec{r}, t)}{\partial \vec{r}^{2}}+Q(\vec{r}, t)
$$

where $T(r, t)[\mathrm{K}]$ is the time $(t)$ and location(radius)-dependent $(r)$ temperature, $Q(r, t)$ $\left[\mathrm{W} / \mathrm{m}^{-3}\right]$ are external and internal heat sources and sinks for example the optical heat excitation of solid surfaces. In general, the parameters $\lambda, \rho$ and $c$ are location and temperature dependent. For homogeneous isotropic materials and small temperature ranges they can be assumed to be constant. The temperature perturbation $\Delta T$ after absorbing a Dirac pulse can be estimated assuming a one-dimensional heat flow within a semi-infinite medium $[8,9]$ with the thermal effusivity $e=\sqrt{\lambda \rho c}$ :

$$
\Delta T=Q / e \sqrt{\pi t}
$$

As described in the Planck's law the surface is emitting infrared radiation dependent of the surface's temperature, the emissivity $\varepsilon$ and the Boltzmann constant. Thus, a transient infrared response of a sample after flash light excitation associated with (5) can be expected to be related to the inner heat conduction processes defined in (4), so that conclusions to the inner properties of solids can be taken.

\subsection{Principals of Pulsed Phase Thermography for Data Acquisition and Evaluation}

Active thermographic testing techniques use defined transient heat flows to characterize materials and samples respectively. After thermal excitation of a sample the temperature distribution is acquired by an infrared detector recording a sequence of thermograms of the object's surface. Following the filtering of the raw data with noise suppression filters [6], further data processing procedures can be conducted.

Pulsed phase thermography (PPT) described in [10] combines advantages of both modulated and pulse methods and has become an important technique for quantitative data analysis [11,12]. This technique enables fast measurements that are quite robust against emissivity changes and other surface inhomogenities and offers a high potential for industrial use. A heat pulse stimulates a burst of thermal waves with frequencies up to a limit that is restricted by its temporal profile. The thermal waves propagate into the material and the sample responds to all incoming frequencies with corresponding amplitudes and phase shifts. The various frequencies the infrared signals are then transferred into the frequency domain by the well known Fourier transform. Computation time is reduced by using the discrete Fast Fourier Transformation (FFT) [12]. An adequate set of acquisition parameter regarding sampling rate and observation time have an enormous influence to the ability to 
detect inner irregularities in pulse phase experiments [13]. Therefore, a thorough adjustment for the present problem is of great importance.

\section{Finite Element Model of the Spot Weld}

\subsection{D-Geometry}

The geometry of the model for the finite element calculations is aligned to a minimum accepted spot weld to assure that the border case is covered. The sheet metal thicknesses are $t_{s}=1.5 \mathrm{~mm}$, the gap between the sheets is $t_{g}=0.3 \mathrm{~mm}$, the lens-diameter $d_{L=} 4.3 \mathrm{~mm}$ and nugget-diameter $d_{N}=5.0 \mathrm{~mm}$ (figure $2 \mathrm{a}$ ). The material parameters for micro-alloyed steel are $\lambda=54 \mathrm{~W} /(\mathrm{m} \cdot \mathrm{K}), c=465 \mathrm{~J} /(\mathrm{kg} \cdot \mathrm{K}), \rho=7.833$ $\mathrm{kg} / \mathrm{m}^{3}$ [6]. Expanded to a 3D-geometry the model with rotation symmetry builds the basic model for the simulations (figure $2 b$ ).

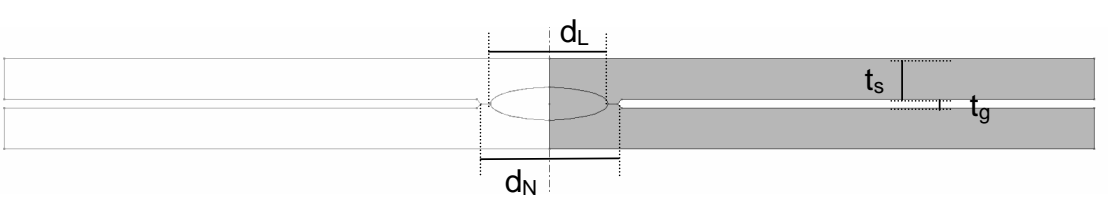

Figure 2a. Definition of the spot weld geometry

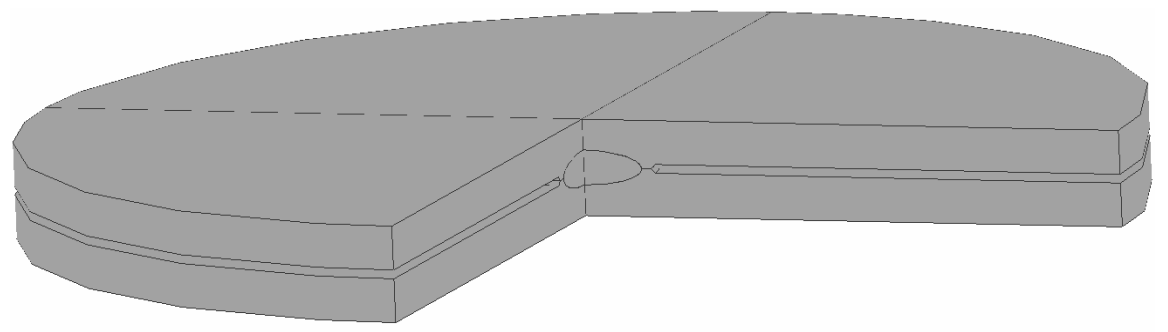

Figure $2 b$. $3 D$ - model of the spot weld as $2 D$ - rotation symmetry of (2a)

\subsection{Modelling Assumptions and Idealizations}

To model the pulsed phase thermography experiment, the heat diffusion equation Eq. (3) must be solved while taking a pulsed heat input into the upper surface into consideration. For the initial conditions room temperature is assumed. The flash light pulse is described by $Q(t)$ with an increase time $T_{\text {increase }}=0.05 \mathrm{~ms}$ and a decrease time $T_{\text {decrease }}=3 \mathrm{~ms}$ qualitatively fitted to manufacturer's specifications and compared with real measurements:

$$
Q(t)=Q_{0} \cdot\left(e^{-\frac{t}{T_{\text {decrease }}}}-e^{-\frac{t}{T_{\text {increase }}}}\right)
$$

Furthermore, assumptions for the boundary conditions are made. The heat loss into the environment can be calculated regarding the occurring radiation and a component described by the heat transfer coefficient $(h)$. However, these losses in 
the measurement will not be crucial, because the heat flux through the spot weld dominates the thermal response of the sample's surface. Inner boundaries are assumed as continuous or described with aid of the heat transfer coefficient $(h)$. The transient temperature distribution of the surface is used directly as simulation data output. This means that the infrared emissivity of the specimen is set to $\varepsilon=1$.

\subsection{Validation of the Finite Element Model}

In a first estimation of the reliability of the simulation results, the finite element model's temperature evolution at several points of the surface is compared to experimental data. While image $3 a$ shows the simulation output, image $3 \mathrm{~b}$ gives the measured data from a sample. The plots show the temperature decays in surface points in the center of the spot weld and the distances of 4,5 and $10 \mathrm{~mm}$ beside it. In the center of the spot weld, the temperature distribution is dominated by the heat flow into the bottom sheet, whereas reflections of the thermal waves at the rear side of the sheet influence the decay in the base material. Between these border cases hybrid phenomena appear, additionally affected by lateral heat flow and further geometry effects.

a) Simulation

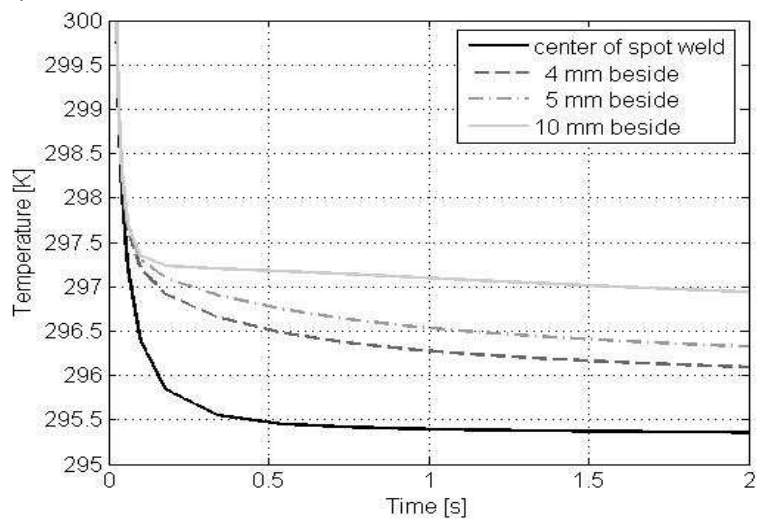

b) Experiment

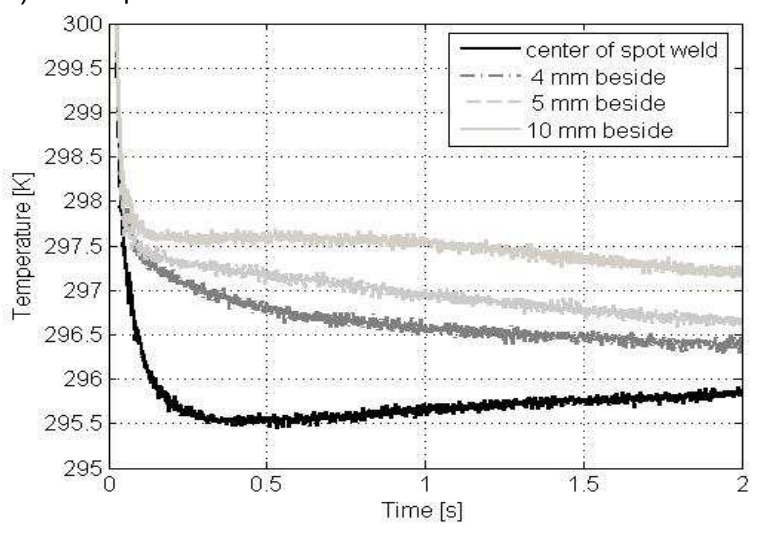

Figure 3. Temperature decays for surface points in different distances to the spotweld center of a) the simulation model and b) a black painted sample 
The decay characteristics and their differences between each other (from one location to another) are responsible for the possibility to evaluate thermal data. Therefore, a congruence of the simulation and the experimental decays is essentially required to receive useful information from the finite element model. The results of the simulation in figure 3 a show a good agreement with the experimental data in figure $3 \mathrm{~b}$ although differences, i.e. the increasing temperature in the center of the spot weld after longer time intervals, need to be discussed in order to enhance the simulation model.

\section{Spot Weld Evaluation}

\subsection{Experimental Setup and Samples}

The experimental setup is equipped with a flash light $(6 \mathrm{~kJ})$ and an infrared camera situated on the same side of the specimen as the excitation source. The sequences are recorded with a highly sensitive semi conductor detector array (NEDT $<20 \mathrm{mK}$ ) with sample rates up to $750 \mathrm{~Hz}$. For sample preparation hot-dip galvanized sheets of the micro-alloyed high-strength steel $\mathrm{HX} 320 \mathrm{LAD}^{1}$ with the thickness of 1.5 $\mathrm{mm}$ each were joined together by resistance spot welding in a configuration with a 16 $\mathrm{mm}$ overlap.

\subsection{Parameter Optimization for Evaluation}

Figure 4 shows an intersection of a characteristic spot weld. Relevant positions or boundaries (i.e. fusion line) for the thermal waves passing through the weld during the measurement are indicated. The paths from top surface to the joint plane $(1.5 \mathrm{~mm})$ and further to the bottom surface $(3.0 \mathrm{~mm})$ are predefined as the most relevant paths for the evaluation of the spot weld diameter.

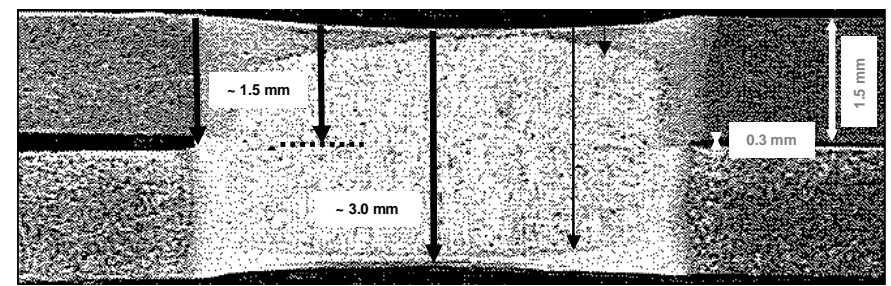

Figure 4. Definitions for the spot and nugget diameter of the spot weld

To receive information from the joint plane or the bottom side, the thermal diffusion lengths (2) should be at least equal to the corresponding path lengths. For the present sample with $\alpha=1.47 \cdot 10^{-5} \mathrm{~m}^{2} / \mathrm{s}$ and with $\mu=\sqrt{2 \alpha / \omega} \Leftrightarrow f=\alpha / \pi \cdot \mu^{2}$ the maximum frequencies are $2 \mathrm{~Hz}$ (for sensitivity in $1.5 \mathrm{~mm}$ depth) and $0.5 \mathrm{~Hz}$ (for sensitivity in $3.0 \mathrm{~mm}$ depth).

Even though low frequencies are required to visualize deep geometry effects, high frequencies are preferred to receive best contrasts in phase shifts. Because the thermal wavelengths Eq. (3) are large compared to the sample's thickness

\footnotetext{
${ }^{1}$ in accordance with DIN EN 10292
} 
( $\lambda \approx 6 \mu \approx 6 t)$, high frequencies for the phase shift analysis are also preferred to obtain adequate sensitivities.

The time range of interest can be narrowed to the interval, where deviations in the temperature decay influenced by the spot weld geometry are expected. Determined by Eq. (1), with $v_{p}=$ distance $/ \mathrm{t}=\sqrt{2 \alpha \omega} \Leftrightarrow t=$ distance $/ 2 \sqrt{\alpha \pi f}$, contrasts caused by reflection in the different depths start to appear after the frequencydependent times listed in table 1. There will be no signal for $2 \mathrm{~Hz}$ and the depth of $3.0 \mathrm{~mm}$ because the thermal diffusion length is limited to $1.5 \mathrm{~mm}$.

Table 1. Relevant points in time for spot weld evaluation in dependence of depth and frequency

\begin{tabular}{|ll|l|}
\hline Depth & frequency & point in time \\
\hline $3.0 \mathrm{~mm}$ & $2.0 \mathrm{~Hz}$ & $0.31 \mathrm{~s}$ \\
$3.0 \mathrm{~mm}$ & $0.5 \mathrm{~Hz}$ & $0.62 \mathrm{~s}$ \\
$1.5 \mathrm{~mm}$ & $2.0 \mathrm{~Hz}$ & $0.16 \mathrm{~s}$ \\
$1.5 \mathrm{~mm}$ & $0.5 \mathrm{~Hz}$ & $0.31 \mathrm{~s}$ \\
\hline
\end{tabular}

However, the selection of suitable analysis parameter is strongly restricted by the experimental setup, particularly by the camera performance and the excitation source. As a first approximation, we assume no disturbance by the flash light excitation after $0.005 \mathrm{~s}$, whereas the frame rates and the recorded time ranges have to be fitted thoroughly to the expected effects estimated by the analytical approach before. With a sampling rate of $750 \mathrm{~Hz}$ the optimized parameters for the Fourier transform result in a total amount of 375 thermograms for $2 \mathrm{~Hz}$ and 1500 thermograms for the $0.5 \mathrm{~Hz}$ analysis. The relevant points in time (table 1) are included in both inspected time ranges. Therefore, the chosen evaluation parameters consider the border cases between the highest required depth resolution and the best accuracy of measurements that is intimately connected with the contrast evolution.

\subsection{Determination of the spot weld diameter}

The pulse phase analysis conducted in order to determine the spot weld sizes is based on the phase shifts caused by the change of sheet thickness in the joint area. In each thickness area, a different phase shift is generated and can be observed by the infrared detector. The phase shifts are extracted from the infrared signal by the aid of Fourier transformations.

The measurement is conducted for two carefully welded joints. The phase shift images of a $2 \mathrm{~Hz}$ and a $0.5 \mathrm{~Hz}$ FFT-analysis with the frame rate of $750 \mathrm{~Hz}$ are illustrated in figure 5 . In a first step, the samples are painted black to get idealized conditions for high quality infrared signals (fig. 5a). In a second step, the bare metallic surface equivalent to production line conditions is equally evaluated (fig. $5 b$ ). Figure $5 c$ shows the results for a selective oil contamination on the sample's surface. 
a)

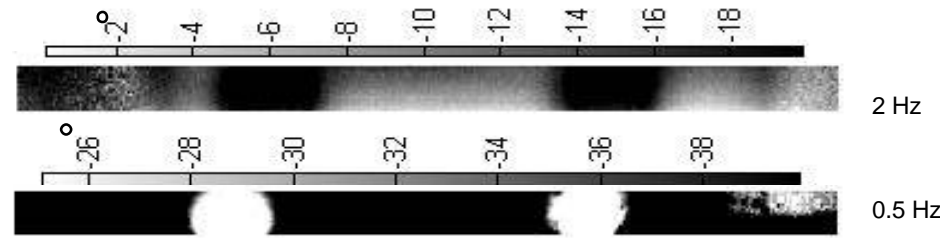

b)
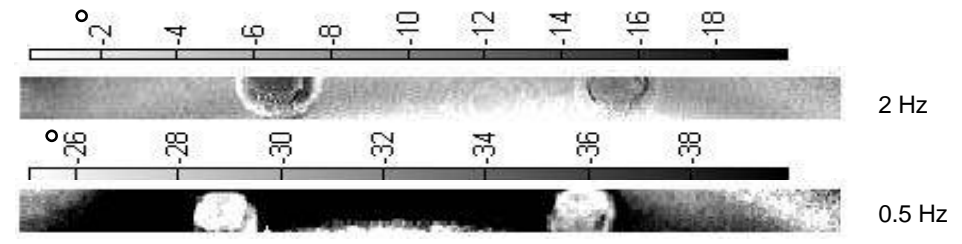

c)

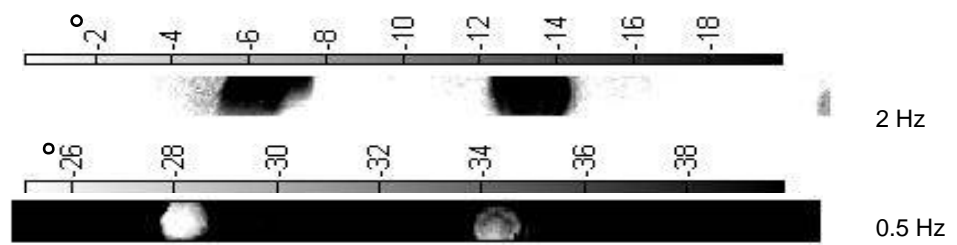

Figure 5. Phase shift images of a) black painted surface, b) bare metallic surface and c) selective oil contamination on the surface

Looking at the result patterns of the black painted sample in figures $5 \mathrm{a}$, two spot welds become clearly visible. The evaluation parameters of the $0.5 \mathrm{~Hz}$ analysis lead to the most homogenous phase contrasts. The determination of thermal diameters by defining thresholds will be the next step. The result images of the measurements with real sample surfaces (fig. 5b) differ remarkably from the previous ones. Especially within the $2 \mathrm{~Hz}$ analysis artefacts occur that indicate surface inhomogenities (e.g. colour variations, electrode indentation). Due to the decreasing sensitivity to surface disturbances, for lower frequencies the absence of the black paint is not as serious as for high frequencies. A further preview of the significance of certain surface disturbances will be given adjacently in figure 5c. The oil contamination on the sample's surface have a significant influence to the $2 \mathrm{~Hz}$ analysis, whereas the $0.5 \mathrm{~Hz}$ analysis shows again an advantage.

Recapitulating, at this first treatment the parameter set of the $0.5 \mathrm{~Hz}$ analysis seems to be most appropriate for the spot weld evaluation. But to qualify this evaluation technique and its parameter set, disturbances on the measurement have to be taken into account more comprehensively.

\section{Analysis of Disturbances for Pulsed Phase Thermography}

To validate a non destructive testing technique for the industrial deployment, the feasibilities and uncertainties of the measurement have to be defined thoroughly. Several disturbances can affect the thermographic measurement and therefore have to be analyzed in detail. The most important possible influences are illustrated in figure 6 . 


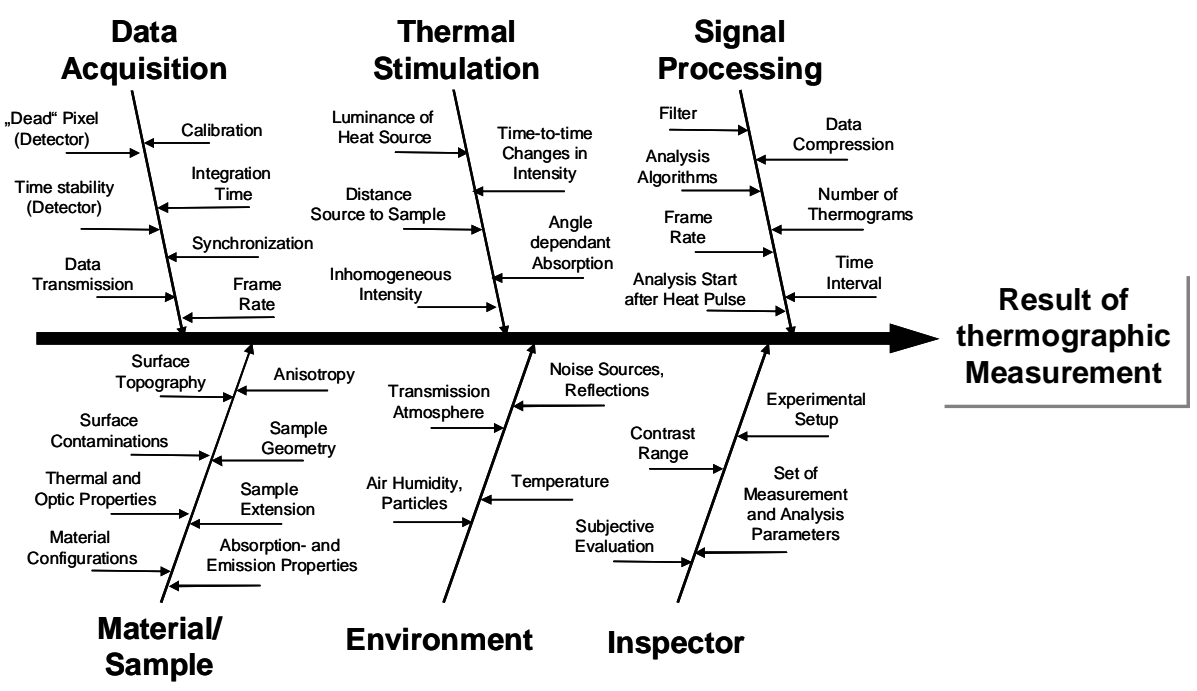

Figure 6. Possible disturbances in thermographic measurements

For the purpose of evaluating the disturbance effects, the finite element simulations demonstrate their capability. Many of the influences can be stated and calculated numerically. For example, the interference of the measured signal caused by emissivity changes in the surface can easily be estimated by varying the (analogous) emissivity parameter $\varepsilon$. Also inhomogenous heat deposition can be described with the aid of the boundary conditions. Even without knowing the exact parameter values, general estimations for worst cases can be made.

\section{Discussion and Future Prospects}

The current investigation presents instruments for the approach to a new testing problem. So the assessment of feasibilities for pulse thermography techniques becomes possible with adequate effort. The non-destructive characterization of resistance spot welds can be validated by the aid of a combination of both experiments and supporting simulations.

Pulse phase thermography experiments were conducted as one option to process thermal data from the experiment: Spot welds were visualized by phase shift images using FFT-analysis of the thermal data. These results indicate the capacity to determine the spot weld diameter and the initial results seem to fulfil the requirements for an industrial application. To consolidate these findings, the influences of all relevant geometry factors (e.g. range of sheet thicknesses, material combinations, surface layers) and the disturbance sources (e.g. surface indentations of the welding electrodes, lateral heat flow effects) have to be carefully investigated. Therefore, the determination of the robustness of the testing technique against typical production disturbances is a matter of further investigations.

The advantage of the finite element simulation is the possibility to study these influences by parameter variations in a very effective way. Worst case situations for 
the implementation can be estimated and a reliable constitution of the feasibilities is possible without larger experimental effort. The simulation data can also be used for a further fitting of the evaluation parameters in the FFT-analysis. Modifications of the processing modes that might be needed in order to suppress disturbances can easily be tested with the simulation data. Consequently, an instrument for the evaluation of even new approaches for data processing is already available.

\section{REFERENCES}

[1] Shepard S., Chaudhry B., Predmesky R., Zaluzec M. Pulsed thermographic inspection of spot welds, SPIE, 3361 (1998), 320-324.

[2] Türler D., Hopkins D., Reverdy, F. Nondestructive Evaluation of Spot Welds Using Acoustic and Thermographic Imaging Techniques, SAE-SP, 1766 (2003), 123-142.

[3] Satonaka S., Matsuyama K. Review on Inspection Techniques for Spot Welds, Welding in the World 44, 3 (2000), 29-36.

[4] Almond D.P., Patel P.M. Phothermal Science and Techniques, Physics and its Applications, 10 (1996)

[5] Rosencwaig A., Gersho A. Theory of the photoacoustic effect in solids, J. Appl. Phys., 47 (1976), 64-9.

[6] Maldague X.P.V. Theory and Practice of Infrared Technology for Nondestructive Testing, John-Wiley \& Sons (2001).

[7] Incropera F.P., DeWitt D.P. Fundamentals of Heat and Mass Transfer, JohnWiley \& Sons 5th edition (2002).

[8] Carslaw H.S., Jaeger J.C. Conduction of heat in solids, Oxford University Press (1959).

[9] Cielo P., Maldague X., Déom A., Lewak R. Thermographic Nondestructive Evaluation of Industrial Materials and Structures, Materials Evaluation, 45 (1987), 452-465.

[10] Maldague X., Marinetti S. Pulse Phase Infrared Thermography, J. Appl. Phys., 79 (1996), 2694-2698.

[11] Ibarra-Castanedo C., González D., Maldague X. Automatic Algorithm for Quantitative Pulsed Phase Thermography Calculations, Proc. 16th World Conference on Nondestructive Testing (WCNDT), 16 (2004).

[12] Maldague X., Galmiche F., Ziadi A. Advances in Pulsed Phase Thermography, Journal of Infrared Physics \& Technology, 43 (2002) 175-181.

[13] Marinetti S., Plotnikov Y., Winfree W., Braggiotti A. Pulse phase thermography for defect detection and visualization, SPIE, 3586 (1999) 230-238. 